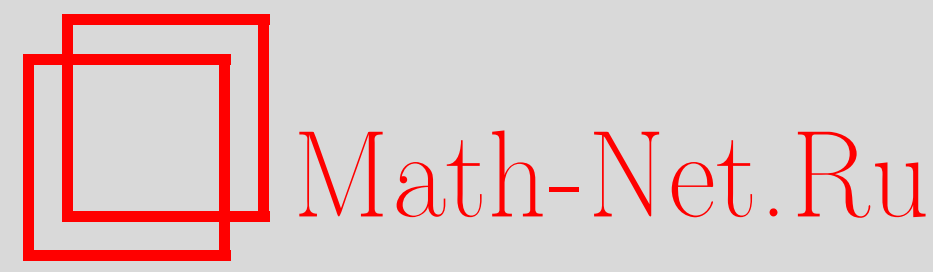

Ю. В. Крякин, О функциях с ограниченной $\mathbf{n}$-й разностью, Изв. РАН. Сер. матем., 1997, том 61, выпуск 2, 95-110

DOI: https://doi.org/10.4213/im117

Использование Общероссийского математического портала Math-Net.Ru подразумевает, что вы прочитали и согласны с пользовательским соглашением

http: //www.mathnet.ru/rus/agreement

Параметры загрузки:

IP : 52.205 .19 .152

26 апреля 2023 г., 17:57:33 
УДК 517.5

\section{Ю.В. Крякин}

\section{О функциях с ограниченной $n$-й разностью}

Найдены точные значения модифицированных констант Уитни для приближения кубическими многочленами. Даны новые оценки констант в случае приближения функции из $C\left(I^{N}\right)$ квазиполиномами.

Библиографиия: 25 наименований.

\section{§1. Введение}

Пусть функция $f(x)$ определена на промежутке $I=[0,1]$. Положим

$$
\begin{aligned}
\Delta_{h}^{n} f(x) & =\sum_{j=0}^{n}(-1)^{j}\left(\begin{array}{l}
n \\
j
\end{array}\right) f(x+j h), \\
\omega_{n}(f) & =\sup _{x, x+n h \in I}\left|\Delta_{h}^{n} f(x)\right| .
\end{aligned}
$$

В 1957 г. Х. Уитни доказал следующий фундаментальньй результат теории приближений.

Tеорема А [1]. Для любой непрерывной на I функции $f(x)$ и натурального $n \geqslant 1$ найдутся алгебраический полином $P_{n-1}(x)$ степени не выше $n-1$ и положительное число $W_{n}$ такие, что

$$
\sup _{x \in I}\left|f(x)-P_{n-1}(x)\right| \leqslant W_{n} \omega_{n}(f) .
$$

Главное внимание в работе [1] было уделено оценке величин $W_{n}$, точные значения которых, следуя Б. Сендову, мы будем назьвать константами Уитни. Их отыскание представляет собой трудную нерешенную задачу. Не существует даже гипотезы об их величине. Поэтому первостепенный интерес представляют оценки констант для конкретных методов приближения. В доказательстве теоремы А сам Уитни применял интерполяционные полиномы по равномерным узлам:

$$
P(i /(n-1))=f(i /(n-1)), \quad i=0, \ldots, n-1 .
$$

Его доказательство было сложным и не позволяло дать оценок констант при всех $n$. Усилия Уитни были сосредоточены на оценках интерполяционных констант $W_{n}^{\prime}$ при малых $n$. Основные результаты работы [1]:

$$
\begin{aligned}
& 1 \leqslant W_{n}^{\prime}, \quad 1 / 2 \leqslant W_{n}<W_{n}^{\prime}<\infty, \\
& \begin{array}{ccccccc}
n & \ldots & 1 & 2 & 3 & 4 & 5
\end{array} \\
& \begin{array}{llllllllll}
W_{n} & \ldots & 1 / 2 & 1 / 2 & 8 / 15 & 7 / 10 & 1 / 2 & 3.25 & 1 / 2 & 10.4
\end{array} \\
& \begin{array}{llllllllll}
W_{n}^{\prime} & \ldots & 1 & 1 & 16 / 15 & 14 / 9 & 1 & 3.25 & 1 & 10.4
\end{array}
\end{aligned}
$$

Работа частично поддержана грантом Международного научного фонда.

$$
\text { (C) Ю.В. Крякин } 1997
$$


Одно значение обозначает точную постоянную, пара - найденные Х. Уитни верхние и нижние гранищы.

Дальнейшее продвижение в получении хороших оценок констант Уитни связано с именем Б. Сендова. Опираясь на компьютерные эксперименты, он в 1982 г. выдвинул гипотезу об ограниченности констант $W_{n}$ единицей и констант $W_{n}^{\prime}$ двойкой [2]. Эти гипотезы выглядели весьма смело, так как существующие к тому времени оценки констант были очень завышены $\left(W_{n}<c n^{2 n}[3]\right)$. Прорыв в данном направлении произошел в 1985 г. В течение одного месяца были опубликованы работы [4]-[6], приведшие к доказательству ограниченности констант Уитни величиной, не зависящей от степени приближающего полинома: $W_{n}<6$ [7]. При этом использовались модифицированные интерполяционные полиномы, удовлетворяюшие условиям

$$
R(i /(n+1))=f(i /(n+1)), \quad i=1, \ldots, n .
$$

Дальнейшее развитие темы таково: $W_{n}<3$ (независимо Б. Сендов [8], Ю. А. Брудньй [9], автор [10]), $W_{n}<2$ (автор [11]).

Доказательства Б. Сендова и Ю. А. Брудного не были опубликованы, автор же использовал для получения оценок многочлены, интерполяционные в среднем:

$$
\int_{0}^{i / n}\left(f(x)-Q_{n-1}(x)\right) d x=0, \quad i=1, \ldots, n
$$

При $n=1,2$ эти полиномы совпадают с полиномами, ранее применявшимися Э. А. Стороженко [12] для доказательства теоремы Уитни в классах $L^{p}, p \geqslant 1$. Использование многочленов $Q_{n-1}$ позволило упростить имеющиеся конструкции и получить новые результаты в близких задачах. Даны новые оценки констант Уитни в интегральной метрике [13], [14], улучшающие оценку Сендова-Такева [15] . Доказано [16], что интерполяционные константы $W_{n}^{\prime}$ не превосходят пятерки. Одной из особенностей применения многочленов $Q_{n-1}$ является простота оценок снизу. Константы для интерполяции в среднем $\widetilde{W}_{n}$ ограничены снизу единицей, и эта оценка точна [11]. Кроме того, оказалось возможным найти значения констант в случае локального приближения многочленами нулевой, первой и второй степеней [17]: $\widetilde{W}_{1}=\widetilde{W}_{2}=\widetilde{W}_{3}=1$.

Главной целью настоящей работы является получение точного неравенства для локального приближения многочленами третьей степени. Таким образом, наиболее сильный результат на сегодняшний день таков.

ТЕОРемА 1. Для любой непрерьвной на I функиии $f(x)$ и натурального $n \geqslant 1$

$$
\begin{gathered}
\sup _{x \in I}\left|f(x)-Q_{n-1}(x)\right| \leqslant \widetilde{W}_{n} \omega_{n}(f), \\
\widetilde{W}_{n} \leqslant\left\{\begin{array}{lll}
2, & \text { если } \quad n \geqslant 5, \\
1, & \text { если } \quad n \leqslant 4 .
\end{array}\right.
\end{gathered}
$$

Случай $n=4$, которому в основном посвящена настоящая работа, технически достаточно сложен, поэтому мы сразу опишем идеи, на которые опирается доказательство. 
Прежде всего скажем несколько слов об асимптотической оценке $\widetilde{W}_{n} \leqslant 2$. В ее основе лежит доказательство, восходящее к А. Берлингу, для случая бесконечного отрезка (см. [1]). Главная идея этого доказательства, встречаюшаяся во всех работах последних лет, может быть охарактеризована словом "усреднение". Многочлены, интерполящионные в среднем, как нам кажется, являются удобным аппаратом для адекватного выражения этой идеи. С другой стороны, на наш взгляд, для получения точных результатов одной этой идеи не достаточно. При получении точных оценок мы использовали дополнительные рассуждения, восходяшие к Уитни и связанные с применением тождеств специального вида. К сожалению, и в работе Уитни, и в нашей работе это сопряжено с большими вычислительньми трудностями, и в данный момент не ясно, удастся ли на этом пути продвинуться дальше. Вероятно, требуются новые соображения, которые пока не найдены.

План работы таков. Схема доказательства основного результата приведена в небольшом $\S 2$. Вспомогательные предложения, нужные для этого, отнесены в $\S 3$. Детали доказательства теоремы 1 для малых $n$ приведены в $§ 4 ; \S 5$ посвящен многомерному обобшению теоремы 1.

\section{§2. Схема доказательства равенства $\widetilde{W}_{n}=1, n<5$}

Существенным моментом доказательства является использование конечных разностей

$$
\Delta_{h_{1}, h_{2}}^{n} F(x, y)=\sum_{j=0}^{n}(-1)^{j}\left(\begin{array}{l}
n \\
j
\end{array}\right) F\left(x+j h_{1}, y+j h_{2}\right)
$$

функции

$$
F(x, y)=(x-y)^{-1} \int_{x}^{y} f(t) d t, \quad F(x, x)=f(x) .
$$

В силу однородности оценки будем считать

$$
\omega_{n}(f) \leqslant 1, \quad F(0, i / n)=0, \quad i=1, \ldots, n .
$$

Для доказательства достаточно предположить, что $f(x)=\sup _{y \in I}|f(y)|>1$, и показать, что это невозможно при условии (1).

Удобно разделить доказательство на два случая:

a) точка максимума $x$ лежит около конща отрезка;

б) точка максимума $x$ отделена от конца отрезка.

Трудным является случай а). Его мы здесь и рассмотрим. Отметим, что в силу симметрии достаточно считать точку $x$ расположенной около левого конца отрезка.

Важное значение в дальнейшем имеет следующая модифищированная разность:

$$
R_{\alpha, h}=\left(f(x)-\Delta_{\alpha x, h}^{n} F(x, x)\right)\left(\frac{-x}{h-\alpha x}\right)^{-1}, \quad h=\frac{1-x}{n}, \quad \alpha<\frac{h}{x} .
$$

Из условия (1) и леммы 1 (см. $\S 3$ ) вытекает неравенство, поэтому $\Delta_{\alpha x, h}^{n} F(x, x) \leqslant 1$. Следовательно,

$$
R_{\alpha, h}<0
$$


Используя лемму 2 из $\S 3$, разложим $R_{\alpha, h}$ на разности малого и большого шага:

$$
\begin{gathered}
R_{\alpha, h}=\alpha r_{x}+r_{h}, \\
r_{x}=f(x)-\Delta_{\alpha x, 0}^{n} F(x, x), \quad r_{h}=\left(f(x)-\Delta_{0, h}^{n} F(x, x)\right)(-h / x) .
\end{gathered}
$$

Разность большого шага в свою очередь, пользуясь леммой 3 , запишем в виде

$$
\begin{gathered}
r_{h}=r_{h, x}+r_{h, h}, \\
r_{h, x}=\sigma_{n} F(0, x), \quad \sigma_{n}=\sum_{i=1}^{n} \frac{1}{i}, \\
r_{h, h}=\sum_{j=1}^{n}(-1)^{j}\left(\begin{array}{c}
n \\
j
\end{array}\right) \frac{n-j}{n j} F\left(\frac{j}{n}, x+j h\right) .
\end{gathered}
$$

Экспоненциальная оценка средних внутри отрезка (лемма 4)

$$
\left|F\left(\frac{i}{n}, \frac{i}{n}+t\right)\right| \leqslant\left(\begin{array}{c}
n \\
i
\end{array}\right)^{-1} \exp \left(n t\left(\sigma_{i}-\sigma_{n-i}\right)\right)
$$

влечет неравенство $\left|r_{h, h}\right| \leqslant \sigma_{n}-1$ (лемма 5). Таким образом, из (2) следует

$$
M_{\alpha}(n)=\alpha r_{x}+r_{h, x}<\sigma_{n}-1 .
$$

Доказательство основано на тождестве

$$
A f(x)=\sum a_{i} f_{i}+\sum b_{j} \Delta_{j}^{n}+\sum c_{k} M_{\alpha(k)}(n),
$$

коэффициенты которого удовлетворяют условиям

$$
A>\sum\left|a_{i}\right|+\sum\left|b_{j}\right|+\left(\sigma_{n}-1\right) \sum c_{k}, \quad c_{k}>0
$$

Здесь и ниже $f_{i}=F\left(\frac{x(i-1)}{2}, \frac{x i}{2}\right)$ - средние значения функции, $\Delta_{j}^{n}-$ средние значения конечных разностей.

Предположения $f_{i}<f(x),-1 \leqslant \Delta_{j}^{n} \leqslant 1<f(x)$ и условия (3), (5) противоречат равенству (4). Это доказывает теорему 1 в случае а).

Конкретный вид тождеств (4) для значений $n=2,3,4$ дан в $\S 4$. Там же приведены комментарии по поводу роли слагаемых в правой части тождества.

\section{§3. Вспомогательные предложения}

Леммы данного параграфа касаются оценок разностей функций $F(x, y)$. Первая из них говорит о том, что разности сглаженных функций “не превосходят" разности исходных функций.

ЛЕмма 1 [11]. Имеем

$$
\Delta_{h_{1}, h_{2}}^{n} F(x, y)=\int_{0}^{1} \Delta_{h_{1}+t\left(h_{2}-h_{1}\right)}^{n} f(x+t(y-x)) d t
$$

Вторая лемма (проекционное свойство разностей) - следствие соответствующего свойства средних значений. 


\section{Лемма 2. Имеем}

$$
\Delta_{u, v}^{n} F(x, x)=\frac{v}{v-u} \Delta_{0, v}^{n} F(x, x)+\frac{u}{u-v} \Delta_{u, 0}^{n} F(x, x) .
$$

ДоКАЗАТЕЛЬСТВО. Воспользуемся следуюшим тождеством для средних $F(x, y)$, которое вытекает непосредственно из определения $F(x, y)$ :

$$
F(x, y)=\frac{z-x}{y-x} F(x, z)+\frac{y-z}{y-x} F(z, y) .
$$

Раскрывая конечную разность и применяя выписанное тождество к функции под знаком суммы, получаем

$$
\begin{aligned}
\Delta_{u, v}^{n} F(x, x) & =\sum_{j=0}^{n}(-1)^{j}\left(\begin{array}{c}
n \\
j
\end{array}\right) F(x+j u, x+j v) \\
& =\sum_{j=0}^{n}(-1)^{j}\left(\begin{array}{c}
n \\
j
\end{array}\right)\left\{\frac{u}{u-v} F(x+j u, x)+\frac{v}{v-u} F(x, x+j v)\right\} \\
& =\frac{v}{v-u} \Delta_{0, v}^{n} F(x, x)+\frac{u}{u-v} \Delta_{u, 0}^{n} F(x, x) .
\end{aligned}
$$

Третья и четвертая леммы - существенная часть вывода асимптотической оценки $\widetilde{W}_{n}<2$. Они и пятая лемма используются для доказательства важного неравенства (3).

Лемма 3 [11]. Пусть $F(0, i / n)=0, \quad i=1, \ldots, n, \quad x=\frac{1-h}{n}, \quad \frac{1}{n+1}<h<\frac{1}{n}$. Тогда

$$
f(x)-\Delta_{0, h}^{n} F(x, x)=\frac{-x}{h}\left\{\sum_{j=1}^{n}(-1)^{j}\left(\begin{array}{l}
n \\
j
\end{array}\right) \frac{n-j}{n j} F\left(\frac{j}{n}, x+j h\right)+\sigma_{n} F(0, x)\right\} .
$$

ЛЕмма 4 [11]. Eсли $F(0, i / n)=0, i=1, \ldots, n, \omega_{n}(f) \leqslant 1,0<t \leqslant 1 / n, m o$

$$
\begin{aligned}
\left|F\left(\frac{i}{n}, \frac{i}{n}+t\right)\right| & \leqslant\left(\begin{array}{c}
n \\
i
\end{array}\right)^{-1} \prod_{j=1}^{i}\left(1+\frac{n t}{j}\right) \prod_{j=1}^{n-i}\left(1-\frac{n t}{j}\right) \\
& \leqslant\left(\begin{array}{c}
n \\
i
\end{array}\right)^{-1} \exp \left(n t\left(\sigma_{i}-\sigma_{n-i}\right)\right) .
\end{aligned}
$$

Лемма 5. Пусть $F(0, i / n)=0, i=1, \ldots, n, x=(1-h) / n$. Тогда

$$
\left|\sum_{j=1}^{n}(-1)^{j}\left(\begin{array}{l}
n \\
j
\end{array}\right) \frac{n-j}{n j} F\left(\frac{j}{n}, x+j h\right)\right| \leqslant \sigma_{n}-1 .
$$


ДоКАЗАТЕЛЬСТвО. Предположим, что $n=2 k+1 \geqslant 3$ (доказательство при $n=2 k$ проводится аналогично). Нетрудно проверить, что $x+j h=j / n+$ $(n-j) x / n$. Поэтому применение леммы 4 дает

$$
\left|\sum_{j=1}^{n}(-1)^{j}\left(\begin{array}{l}
n \\
j
\end{array}\right) \frac{n-j}{n j} F\left(\frac{j}{n}, x+j h\right)\right| \leqslant \sum_{j=1}^{[(n-1) / 2]} y_{j}(x),
$$

где

$$
y_{j}(x)=\frac{n-j}{n j} e^{-c_{j} x}+\frac{j}{n(n-j)} e^{c_{j} x}, \quad c_{j}=j\left(\sigma_{n-j}-\sigma_{j}\right) .
$$

Нетрудно проверить, что $y_{j}^{\prime}(x)<0$ при $e^{c_{j} x}<(n-j) / j$. Справедливость последнего неравенства следует из оценок

$$
\sigma_{n-j}-\sigma_{j} \leqslant 2 \ln \left(\frac{n-j}{j}\right), \quad j<\frac{n}{2}, \quad x<\frac{1}{n} .
$$

Таким образом,

$$
\sum_{j=1}^{[(n-1) / 2]} y_{j}(x) \leqslant \sum_{j=1}^{[(n-1) / 2]} y_{j}(0)=\sigma_{n}-1
$$

Для получения явного вида функций $M_{\alpha}(n)$ нам понадобится

Лемма 6. Имеем

$$
M_{\alpha}(n)=\alpha \sum_{j=1}^{n} F(x+\alpha(j-1) x, x+\alpha j x) m_{j}(n)+\sigma_{n} F(0, x),
$$

əде

$$
m_{i}(n)=\sum_{k=i}^{n}(-1)^{k+1}\left(\begin{array}{l}
n \\
k
\end{array}\right) \frac{1}{k}
$$

ДокАЗАТЕЛЬСтво. Непосредственно из определения (см. (3)) вытекает

$$
M_{\alpha}(n)=\alpha r_{x}+r_{h, x}=\alpha\left(f(x)-\Delta_{\alpha x, 0}^{n} F(x, x)\right)+\sigma_{n} F(0, x) .
$$

Нетрудно проверить, что первое слагаемое равно

$$
\begin{aligned}
& \alpha \sum_{j=1}^{n}(-1)^{j+1}\left(\begin{array}{c}
n \\
j
\end{array}\right) F(x(1+\alpha j), x) \\
& \quad=\alpha \sum_{j=1}^{n}(-1)^{j+1}\left(\begin{array}{c}
n \\
j
\end{array}\right) \frac{1}{j} \sum_{k=1}^{j} F(x+\alpha(k-1) x, x+\alpha k x) \\
& \quad=\alpha \sum_{j=1}^{n} F(x+\alpha(j-1) x, x+\alpha j x) m_{j}(n) .
\end{aligned}
$$




\section{§4. Доказательство основного результата}

\section{1. Доказательство основного результата в случае точки максиму-} ма около левого конца отрезка. В данном пункте приведены конкретные тождества типа (4). Их вид сильно усложняется при переходе от $n=2$ к $n=4$. Идею получения этих тождеств и их смысл удобно проследить, начав со сравнительно простого случая $n=2$.

ФОРМУЛЫ ДЛЯ КОНЕчНЫХ РАЗНОСТЕЙ. СЛУчАЙ $n=2$. Для $x \in[0,1 / 3]$

$$
f(x)=\frac{1}{12}\left(f_{5}+f_{6}\right)-\frac{1}{2} \Delta_{x, 0}^{2} F(0, x)+\frac{1}{3} M_{1}(2) .
$$

КомменТАРИй. Величина

$$
\Delta_{x, 0}^{2} F(0, x)=\frac{1}{2}\left(f_{1}+f_{2}+f_{3}+f_{4}\right)-2 f(x)
$$

служит для создания в правой части значения $f(x)$. Дополнительные слагаемые $f_{1}, f_{2}, f_{3}, f_{4}$ уничтожаются с помошью функции

$$
M_{1}(2)=f(x)-\Delta_{x, 0}^{2} F(x, x)+\sigma_{2} F(0, x)=\frac{3}{4}\left(f_{1}+f_{2}+f_{3}+f_{4}\right)-\frac{1}{4}\left(f_{5}+f_{6}\right) .
$$

Выполнение условий (5) очевидно.

ФОРМУЛЫ ДЛЯ КОНЕЧНЫХ РАЗНОСТЕЙ. СлУЧАЙ $n=3$. Для $x \in[0,1 / 6]$, $f_{0}=f(0)$

$$
\begin{gathered}
A f(x)=\sum_{i=0}^{12} a_{i} f_{i}+\sum_{j=1}^{4} b_{j} \Delta_{j}^{3}+c M_{1}(3), \\
A=56.571, \quad c=12, \\
a=[4 / 3 ; 0 ; 0 ; 0 ; 21.143 ; 0.714 ; 0.714,0 ; 0 ; 0 ;-0.444 ;-0.444 ;-0.444], \\
b=[-22 / 7 ; 22 / 7 ;-88 / 7 ;-4 / 3], \\
\Delta_{1}^{3}=\Delta_{x / 2,0}^{3} F(x / 2, x), \quad \Delta_{2}^{3}=\Delta_{x / 2,0}^{3} F(0, x), \\
\Delta_{3}^{3}=\Delta_{x, x / 2}^{3} F(0, x / 2), \quad \Delta_{4}^{3}=\Delta_{2 x, 3 x / 2}^{3} F(0,0) .
\end{gathered}
$$

КомментАРий. Существенным в формуле является выбор конечных разностей. Их узлы изображены на рис. 1.

Интерпретация функций разностей такова: $\Delta_{1}^{3}, \Delta_{2}^{3}, \Delta_{3}^{3}$ нужны для создания доминируюшего коэффициента у $f(x)$. Величины $\Delta_{4}^{3}, M_{1}(3)$ "отвечают" за погашение возникших средних значений.

ЗАмечаниЕ. Другая форма вывода теоремы 1 для $n=3$ дана в [17]. Приведенные здесь доказательства короче и служат введением к случаю $n=4$. 


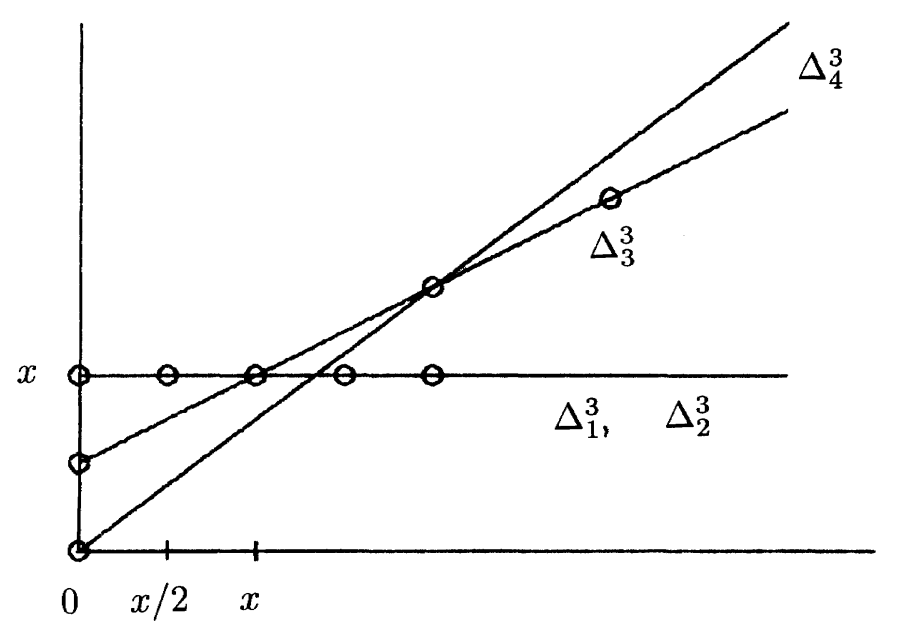

Рис. 1

ФОРМУЛЫ ДЛЯ КОНЕЧНЫХ РАЗНОСТЕЙ. СЛУЧАЙ $n=4$. ДЛя $x \in[0,1 / 12]$

$$
\begin{gathered}
A f(x)=\sum_{i=1}^{22} a_{i} f_{i}+\sum_{j=1}^{12} b_{j} \Delta_{j}^{4}+\sum_{k=1}^{4} c_{k} M_{(k+1) / 2}(4), \\
A=1348, \\
a=[-0.02 ; 0.02 ; 313.37 ; 309.91 ; 0 ; 0.09 ;-0.04 ; 0.09 ; 3.53 ;-0.18 ;-0.03 ; 0.03 ; \\
-12.78 ;-16.72 ; 0.01 ; 0 ;-0.82 ;-0.012 ; 1.69 ; 0.87 ; 1.69 ; 2.51], \\
b=[103.55 ;-181.61 ;-34.84 ;-47.58 ;-1.90 ; 36.60 ; \\
-36.81 ; 9.08 ;-4.09 ; 2.52 ;-0.82 ; 0.82], \\
c=24 \cdot[1.549 ; 5.382 ; 1.073 ; 0.563], \\
\Delta_{1}^{4}=\Delta_{x / 2,0}^{4} F(0, x), \quad \Delta_{2}^{4}=\Delta_{x, x / 2}^{4} F(0, x / 2), \\
\Delta_{3}^{4}=\Delta_{x / 2, x / 2}^{4} F(x / 2,0), \quad \Delta_{4}^{4}=\Delta_{x, x}^{4} F(x / 2,0), \quad \Delta_{5}^{4}=\Delta_{3 x / 2,3 x / 2} F(x / 2,0), \\
\Delta_{6}^{4}=\Delta_{x / 2, x / 2}^{4} F(x, x / 2), \quad \Delta_{7}^{4}=\Delta_{x, x}^{4} F(x, x / 2), \quad \Delta_{8}^{4}=\Delta_{3 x / 2,3 x / 2} F(x, x / 2), \\
\Delta_{9}^{4}=\Delta_{x / 2, x / 2}^{4} F(7 x / 2,3 x), \\
\Delta_{10}^{4}=\Delta_{x / 2, x / 2}^{4} F(10 x, 19 x / 2), \quad \Delta_{11}^{4}=\Delta_{x, x}^{4} F(10 x, 19 x / 2), \\
\Delta_{12}^{4}=\Delta_{x / 2, x / 2}^{4} F(16 x, 31 x / 2) .
\end{gathered}
$$

КомменТАРИй. Усложнение формулы по сравнению с $n=3$ бросается в глаза. Используются четыре функции $M_{\alpha}(n)$, кроме того увеличено число конечных разностей. Их теперь двенадцать. Пути разделенных разностей показаны на рис. 2.

Выбор направлений разностей, увеличение числа и выбор величин $M_{\alpha}(n)$ представляли главную трудность, которую пришлось преодолевать при переходе от значения $n=3$ к значению $n=4$. Сушность возникающей задачи, как нам кажется, прояснится, если мы рассмотрим ее в двойственных терминах, в терминах 


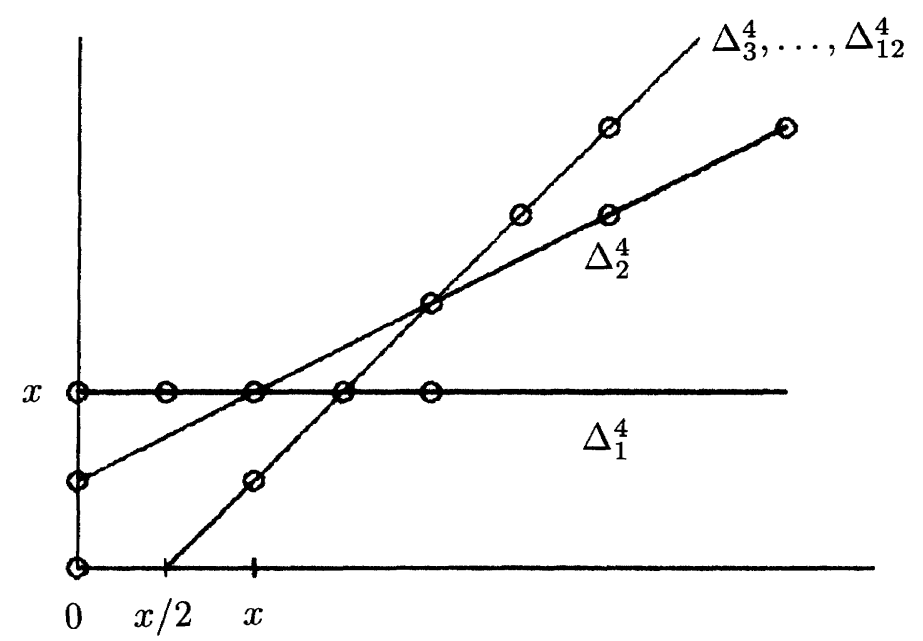

Рис. 2

коэфффищиентов разностей. Используя обычные обозначения

$$
f * g=\int_{-\infty}^{\infty} f(x) g(x) d x
$$

запишем функции $\Delta_{j}^{n}, M_{\alpha}$ в виде

$$
\Delta_{j}^{n}=\widetilde{\Delta}_{j}^{n} * f, \quad M_{\alpha}=\widetilde{M}_{\alpha}(n) * f(x) .
$$

Тождество (4) можно рассматривать теперь как "хорошее" приближение дельта-функции точки $x$ функциями $\widetilde{\Delta}_{j}^{n}, \widetilde{M}_{\alpha}(n)$. Выпишем величины $\widetilde{\Delta}_{j}^{n}, \widetilde{M}_{\alpha}$ в явном виде. Лемма 6 дает

$$
M_{\alpha}(n)=\alpha \sum_{j=1}^{n} F(x+\alpha(j-1) x, x+\alpha j x) m_{j}(n)+\sigma_{n} F(0, x) .
$$

Поэтому

$$
\begin{gathered}
\widetilde{M}_{\alpha}(n)=\widetilde{r}_{x}\left(\frac{t-x}{2 \alpha}\right)+\widetilde{r}_{x, h}(t) \\
\widetilde{r}_{x}(t)=\frac{1}{x} \sum_{i=1}^{n} m_{i}(n) \chi_{i}(t), \quad \widetilde{r}_{x, h}(t)=\frac{1}{x}\left(\chi_{1}(t)+\chi_{2}(t)\right) \sigma_{n}
\end{gathered}
$$

где $\chi_{i}(t)$ - характеристическая функция интервала $I_{i}=(x(i-1) / 2, x i / 2), m_{i}(n)=$ $\sum_{k=i}^{n}(-1)^{k+1}\left(\begin{array}{l}n \\ k\end{array}\right) 1 / k$.

Аналогично,

$$
\widetilde{\Delta}_{j}^{n}=\frac{2}{x} \sum_{i=1}^{m} d_{i j}(n) \chi_{i}(t)+d_{0, j} \delta(x) .
$$

При $n=4$ матрица $d$ имеет вид 


$$
\left(\begin{array}{cccccccccccc}
6 & -4 & 0 & 0 & 0 & 0 & 0 & 0 & 0 & 0 & 0 & 0 \\
0.5 & 1 & 1 & 1 & 1 & 0 & 0 & 0 & 0 & 0 & 0 & 0 \\
-3.5 & 0 & 4 & 0 & 0 & 1 & 1 & 1 & 0 & 0 & 0 & 0 \\
-3.5 & 0 & 6 & -4 & 0 & -4 & 0 & 0 & 0 & 0 & 0 & 0 \\
0.5 & 6 & -4 & 0 & -4 & 6 & -4 & 0 & 0 & 0 & 0 & 0 \\
0 & -2 & 1 & 6 & 0 & -4 & 0 & -4 & 0 & 0 & 0 & 0 \\
0 & -1.667 & 0 & 0 & 0 & 1 & 6 & 0 & 0 & 0 & 0 & 0 \\
0 & 0.333 & 0 & -4 & 6 & 0 & 0 & 0 & 1 & 0 & 0 & 0 \\
0 & 0.333 & 0 & 0 & 0 & 0 & -4 & 6 & 0 & 0 & 0 & 0 \\
0 & 0 & 0 & 1 & 0 & 0 & 0 & 0 & -4 & 0 & 0 & 0 \\
0 & 0 & 0 & 0 & -4 & 0 & 1 & 0 & 0 & 1 & 1 & 0 \\
0 & 0 & 0 & 0 & 0 & 0 & 0 & -4 & 6 & -4 & 0 & 0 \\
0 & 0 & 0 & 0 & 0 & 0 & 0 & 0 & 0 & 6 & 0 & 0 \\
0 & 0 & 0 & 0 & 1 & 0 & 0 & 0 & -4 & -4 & -4 & 0 \\
0 & 0 & 0 & 0 & 0 & 0 & 0 & 1 & 0 & 1 & 0 & 0 \\
0 & 0 & 0 & 0 & 0 & 0 & 0 & 0 & 1 & 0 & 0 & 0 \\
0 & 0 & 0 & 0 & 0 & 0 & 0 & 0 & 0 & 0 & 6 & 1 \\
0 & 0 & 0 & 0 & 0 & 0 & 0 & 0 & 0 & 0 & 0 & -4 \\
0 & 0 & 0 & 0 & 0 & 0 & 0 & 0 & 0 & 0 & 0 & 6 \\
0 & 0 & 0 & 0 & 0 & 0 & 0 & 0 & 0 & 0 & -4 & -4 \\
0 & 0 & 0 & 0 & 0 & 0 & 0 & 0 & 0 & 0 & 0 & 1 \\
0 & 0 & 0 & 0 & 0 & 0 & 0 & 0 & 0 & 0 & 0 & 0 \\
0 & 0 & 0 & 0 & 0 & 0 & 0 & 0 & 0 & 0 & 1 & 0
\end{array}\right)
$$

Вернемся теперь к тождеству (4) и укажем на роли его различных частей. В основном тождестве

$$
A \delta(x)=\sum a_{i} \chi_{i}+\sum b_{j} \widetilde{\Delta}_{j}^{4}+\sum c_{k} \widetilde{M}_{\alpha(k)}(4) .
$$

Сумма $S_{1}=\sum_{j=1}^{2} b_{j} \widetilde{\Delta}_{j}^{4}$ создает большой коэффициент у дельта-функции $\delta(x)$ в правой части; сумма $S_{2}=\sum_{j=3}^{12} b_{j} \widetilde{\Delta}_{j}^{4}$ сглаживает возникаюшие дополнительные слагаемые; сумма $S_{3}=\sum c_{k} \widetilde{M}_{\alpha(k)}(4)$ гасит дополнительные слагаемые; величина $\sum a_{i} \chi_{i}$ характеризует ошибку приближения дельта-функции “коэффициентами конечных разностей”. Сказанное иллюстрируют рис. $3-5$.

4.2. Доказательство основного результата в случае точки максимума, отделенной от конца отрезка. Прежде всего отметим, что достаточно ограничиться предположением $x \in[0,1 / n]$. Для $x \in[1 / n, 1-1 / n]$ доказательство получено в [11].

Основой дальнейших рассуждений является лемма 4.

i) Начнем с простейшего значения $n=2$. Предполагая, что $\max |f(y)|=$ $f(x)>1, x \in[1 / 3,1 / 2], \omega(f) \leqslant 1$, из тождества

$$
\Delta_{0, x}^{2} F(x, 0)=2 F(0,2 x)-2 f(x)
$$

с учетом того, что $\left|\Delta_{0, x}^{2} F(x, 0)\right| \leqslant 1$ (лемма 1$)$, получаем

$$
F(0,2 x) \geqslant \frac{1}{2} f(x)>\frac{1}{2} .
$$




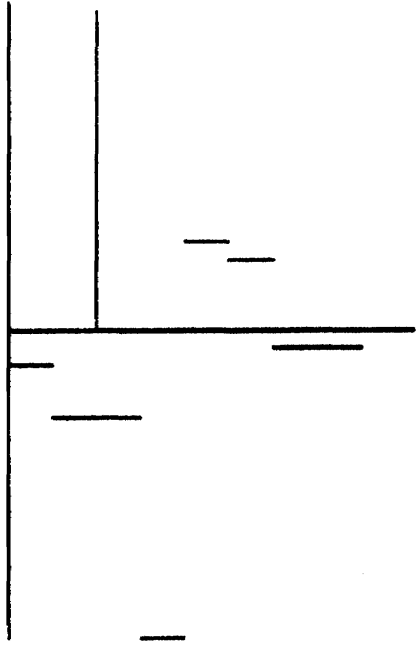

Рис. 3. Сумма $S_{1}$

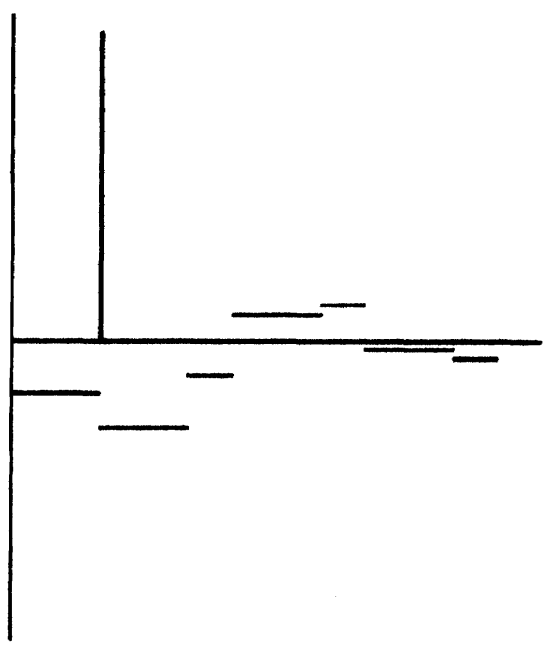

Рис. 4. Сумма $S_{1}+S_{2}$

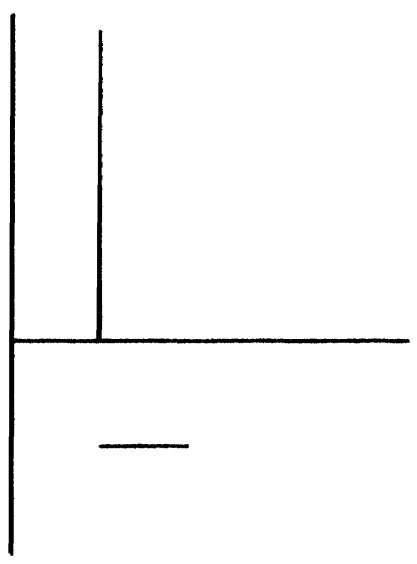

Рис. 5. Сумма $S_{1}+S_{2}+S_{3}$

С другой стороны, принимая во внимание равенство $F(0,1 / 2)=0$ и условие $x \in[1 / 3,1 / 2]$, имеем $F(0,2 x) \leqslant F(1 / 2,2 x)$. Применение леммы 5 дает

$$
F\left(\frac{1}{2}, 2 x\right)=F\left(\frac{1}{2}, \frac{1}{2}+t\right) \leqslant \frac{1}{2}(1+2 t)(1-2 t)<\frac{1}{2},
$$

что противоречит (6).

ii) Рассуждения при $n=3$ проводятся аналогично. Предположим, что $\max |f(y)|=f(x)>1, \quad x \in[1 / 6,1 / 3]$. Ограниченность модуля разностей $\Delta_{0, x}^{3} F(x, 0)$ единицей (лемма 1$)$ и оценка для средних значений леммы 4 приводят к противоречию. Действительно, из леммы 1 следует

$$
\Delta_{0, x}^{3} F(x, 0)=F(x, 0)-3 f(x)+3 F(x, 2 x)-F(x, 3 x) \geqslant-1 .
$$




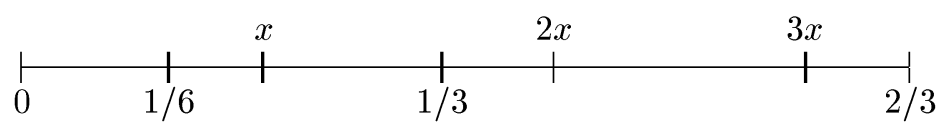

Рис. 6

Оценим слагаемые в данном неравенстве, пользуясь леммой 4. Среднее по интервалу $(0, x)$ не превосходит $(1-3 x)(1-3 x / 2)(1-x)<1 / 3$.

Для среднего по интервалу $(x, 2 x)$ справедлива оценка

$$
|F(x, 2 x)| \leqslant \max (|F(x, 1 / 3)|,|F(1 / 3,2 x)|) .
$$

Средние по интервалам $(x, 1 / 3),(1 / 3,2 x)$ оцениваются с помошью леммы 4 :

$$
\begin{aligned}
\left|F\left(x, \frac{1}{3}\right)\right| & <\frac{1}{3}(1+t)(1-t)\left(1-\frac{t}{2}\right)<\frac{1}{3} \\
\left|F\left(\frac{1}{3}, 2 x\right)\right| & <\frac{1}{3}(1+t)\left(1+\frac{t}{2}\right)(1-t)<\frac{5}{12} .
\end{aligned}
$$

Таким образом, $|F(x, 2 x)|<5 / 12$. Точно такая оценка имеет место и для величины $F(x, 3 x)$. Полученные неравенства противоречат условию $(7)$.

iii) При $n=4, x \in[1 / 12,1 / 4]$ доказательство сложнее. Рассмотрим две возможности:

a) $x \in[1 / 8,1 / 4]$; б) $x \in[1 / 12,1 / 8]$.

a) Ограниченность единищей модуля $\Delta_{x / 2,0}^{4} F(0, x)$ дает

$$
\frac{1}{2} f_{1}-\frac{7}{2}\left(f_{2}+f_{3}\right)+6 f(x)+\frac{1}{2} f_{4} \leqslant 1 .
$$

Так как $f_{1}, f_{4}$ не превосходят $\max |f(y)|=f(x)>1$, то для получения противоречия с (8) достаточно доказать следующую лемму.

ЛЕмма 7. В сделанных предположсениях $\left|f_{2}+f_{3}\right|<1$.

ДокаЗАтельство. Рассмотрим два случая: 1) $x \in[1 / 8,1 / 6]$; 2) $x \in[1 / 6,1 / 4]$.

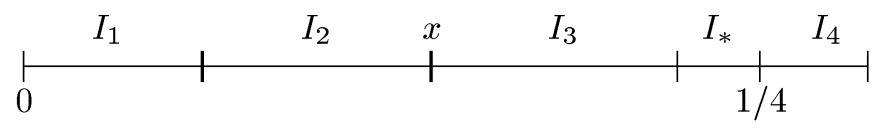

Рис. 7. $x \in[1 / 8,1 / 6]$

В случае 1) (рис. 7 ) доказательство следует из условия $F(0,1 / 4)=0$, которое можно записать в виде $f_{1}+f_{2}+f_{3}+f_{*}=0$, и следующих следствий леммы 4 :

$$
\left|f_{1}\right|<\exp \left(-\frac{1}{4} \sigma_{4}\right)<0.65, \quad\left|f_{*}\right|<\frac{1}{4} \exp \left(\frac{1}{4}\left(\sigma_{3}-\sigma_{1}\right)\right)<0.35 \text {. }
$$


В случае 2) доказательство аналогично.

б) Пусть $x \in[1 / 12,1 / 8]$. Здесь доказательство напоминает доказательство при $x$ около конца отрезка. Решающими являются тождество

$$
\begin{gathered}
A f(x)=\sum_{i=1}^{15} a_{i} f_{i}+\sum_{j=1}^{10} b_{j} \Delta_{j}^{4}, \\
A=29.63, \\
\mathbf{a}=[7.72 ; 5.91 ; 5.71 ; 5.13 ; 4.27 ; 5.41 ;-1.56 ;-0.47 ; \\
0.08 ;-2.52 ;-0.02 ; 0.66 ;-0.9 ; 0.92 ; 0.61], \\
\mathbf{b}=[2.31 ;-3.94 ;-1.31 ;-2.52 ;-1.1 ;-0.04 ;-1.99 ;-1.03 ;-0.61 ; 0.11],
\end{gathered}
$$

условия

$$
f(x)>f_{i}, \quad\left|\Delta_{i}^{4}\right| \leqslant 1
$$

и следующая

ЛЕмМа 8. Имеем

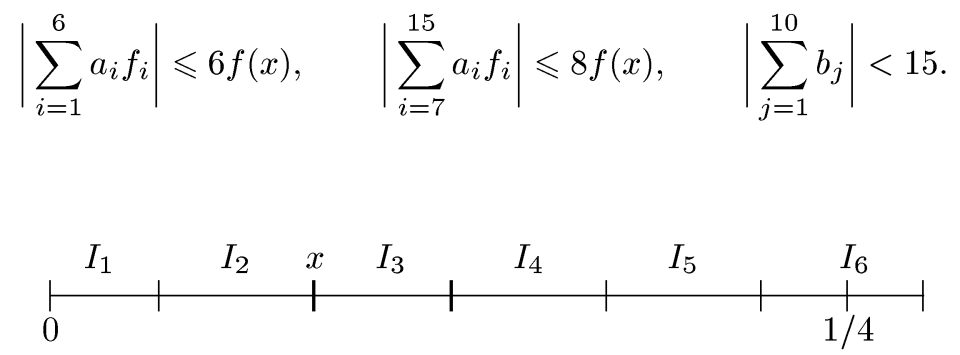

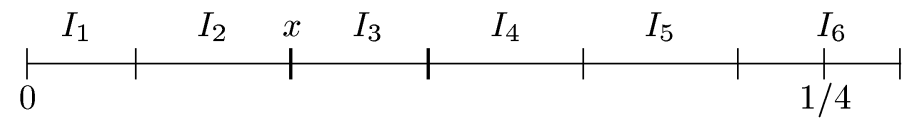

Рис. 8. $1 / 4 \in I_{6}$

ДокАЗАтЕльство. Предположим вначале, что $x \in[1 / 12,1 / 10]$. Тогда $1 / 4 \in$ $I_{6}$ (рис. 8). Условие $\int_{0}^{1 / 4} f(x) d x=0$ позволяет записать выражение $\sum_{i=1}^{6} a_{i} f_{i}$ в виде

$$
\sum_{i=1}^{6} a_{i} f_{i}=\sum_{i=1}^{5} a_{i}^{*} f_{i}+\text { hvost }
$$

где $\mathbf{a}^{*}=\mathbf{a}-\mathbf{5 . 7}=[2.02 ; 0.21 ; 0.01 ;-0.57 ;-1.43] ;$ величина

$$
\text { hvost }=a_{6} f_{6}-5.7 \frac{2}{x} \int_{5 x / 2}^{1 / 4} f(t) d t
$$

не превосходит по модулю

$$
0.29 \frac{2}{x}\left|\int_{5 x / 2}^{1 / 4} f(t) d t\right|+5.41 \frac{2}{x}\left|\int_{1 / 4}^{2 x} f(t) d t\right| .
$$


Применение леммы 4 к каждому из слагаемых дает

$$
\text { hvost }<0.29 \cdot \frac{1}{2}+5.41 \cdot \frac{1}{4}<1.5 \text {. }
$$

Так как $\sum_{i=1}^{5}\left|a_{i}^{*}\right|+1.5<6$, то первое неравенство леммы доказано. Второе неравенство вытекает из оценок $\sum_{i=7}^{15}\left|a_{i}\right|<8,\left|f_{i}\right|<f(x)$, третье неравенство очевидно.

Доказательство при $x \in[1 / 10,1 / 8], 1 / 4 \in I_{5}$ проводится аналогично.

\section{§5. Приложение. Приближение квазиполиномами}

Данный параграфф посвящен переносу теоремы 1 на многомерный случай. Впервые многомерные обобщения теоремы Уитни появились в работах [18], [19]. Доказательства проводились как в классах $C$, так и в $L^{p}, p \geqslant 1$. Случай пространств $L^{p}\left(I^{N}\right)$ был исследован Э. А. Стороженко [20], [21]. Доказательства теоремы в $L^{p}$, $0<p<1$, основанные на других идеях, предложены В. Поповым и П. Петрушевым [22], Э.А. Стороженко и автором [23]. В работах Б. Сендова и М. Такева [24], [25] было доказано, что в случае приближения квазиполиномами в $C\left(I^{N}\right)$ константы не зависят от степени приближающего многочлена. Здесь мы улучшаем оценки Сендова-Такева и получаем точные результаты для приближения квазиполиномами степени не вьше 4.

Пусть $\mathbb{R}^{N}-N$-мерное евклидово пространство, $I^{N}=[0,1]^{N}$ - единичньй куб в нем. Элементы $\mathbb{R}^{N}$ будем обозначать $x=\left(x_{1}, \ldots, x_{N}\right), y=\left(y_{1}, \ldots, y_{N}\right), \ldots$ и писать $x \leqslant y$, если соответствуюшие неравенства выполняются покоординатно; под $x \cdot y$ подразумевается вектор с координатами $x_{i} y_{i}$.

Положим

$$
P_{n}(x)=\sum_{i=0}^{n_{1}} f_{1, i}\left(\hat{x}_{1}\right) x_{1}^{i}+\cdots+\sum_{i=0}^{n_{N}} f_{N, i}\left(\hat{x}_{N}\right) x_{N}^{i},
$$

где $f_{k, i}\left(\hat{x}_{j}\right)$ - непрерьвная функция переменных $x_{1}, \ldots, x_{j-1}, x_{j+1}, \ldots, x_{N}$;

$$
\Delta_{h}^{n} f(\mathbf{x})=\Delta_{h_{1} e_{1}}^{n_{1}} \ldots \Delta_{h_{N} e_{N}}^{n_{N}} f(\mathbf{x}),
$$

где $e_{i}$ - единичньй координатньй вектор.

Для непрерывной в $I^{N}$ функции обозначим через $Q_{n-1}$ квазиполином, удовлетворяюший условиям

$$
\int_{j / n_{i}}^{(j+1) / n_{i}}\left(f(\mathbf{x})-Q_{n-1}(\mathbf{x})\right) d x_{i}=0, \quad i=1, \ldots, N, \quad j=0, \ldots, n_{i}-1 .
$$

Конструктивное определение $Q_{n-1}$ дано в [24], [25].

Teоpema 2. Для $f \in C\left(I^{N}\right)$

$$
\sup _{\mathbf{x} \in I^{N}}\left|f(\mathbf{x})-Q_{n-1}(\mathbf{x})\right| \leqslant \widetilde{W}_{n}(N) \sup _{\mathbf{x}, \mathbf{x}+\mathbf{n} \cdot \mathbf{h} \in I^{N}}\left|\Delta_{h}^{n} f(x)\right|
$$

$c$

$$
\widetilde{W}_{n}(N) \leqslant \widetilde{W}_{n_{1}} \cdots \widetilde{W}_{n_{N}} \leqslant\left\{\begin{array}{lll}
2^{N}, & \text { если } \quad \mathbf{n} \geqslant \mathbf{5} \\
1, & \text { если } \quad \mathbf{n} \leqslant \mathbf{4}
\end{array}\right.
$$


ДоКАЗАТЕЛЬСТВО проводится итерацией одномерного результата, методом математической индукции. Предположим, что теорема справедлива в $\mathbb{R}^{N-1}$. Полагая $g=f-Q_{n-1}$ и учитывая, что $\Delta_{\mathbf{h}}^{\mathbf{n}} f(\mathbf{x})=\Delta_{\mathbf{h}}^{\mathbf{n}} g(\mathbf{x})$, получаем

$$
\sup _{\mathbf{x}}|g(\mathbf{x})|=g\left(\hat{x}_{N}^{0}, x_{N}^{0}\right) \leqslant \widetilde{W}_{n_{2}}\left|\Delta_{h_{N}^{*} e_{N}}^{n_{N}} g\left(\hat{x}_{N}^{0}, x_{N}^{*}\right)\right|
$$

Опираясь на предположение индукции, заканчиваем доказательство:

$$
\begin{aligned}
\sup _{\hat{x}_{N}}\left|\Delta_{h_{N}^{*} e_{N}}^{n_{N}} g\left(\hat{x}_{N}, x_{N}^{*}\right)\right| & =\sup _{\hat{x}_{N}}\left|G\left(\hat{x}_{N}, x_{N}^{*}\right)\right| \\
& \leqslant \widetilde{W}_{n_{1}} \ldots \widetilde{W}_{n_{N-1}}\left|\Delta_{h_{1}^{*} e_{1}}^{n_{1}} \ldots \Delta_{h_{N-1}^{*} e_{N-1}}^{n_{N-1}} G\left(\hat{x}_{N}^{*}, x_{N}^{*}\right)\right| \\
& =\widetilde{W}_{n_{1}} \ldots \widetilde{W}_{n_{N-1}}\left|\Delta_{\mathbf{h}^{*}}^{\mathbf{n}} f\left(\mathbf{x}^{*}\right)\right| .
\end{aligned}
$$

Теорема 2 уточняет результаты Ю.А. Брудного [19] $\left(W_{n}<n^{N n}\right)$, Б. Сендова и М. Такева [24] $\left(W_{n}(2)<49\right)$, М. Такева [25] $\left(W_{n}(N)<N ! 6^{N}\right)$. Так же, как и в одномерном случае, нетрудно показать, что константы теоремы 2 при $\mathbf{n} \leqslant \mathbf{4}$ точны. Пример дает сглаженная дельта-функция вершины куба $I^{N}$. Независимость констант Уитни от размерности пространства при $\mathbf{n} \geqslant \mathbf{5}-$ нерешенная задача. Также не исследован случай приближения функций нескольких переменных полиномами. На наш взгляд, доказательство многомерной теоремы Уитни для полиномов с хорошими оценками констант, например, не зависящими от степени приближающего полинома, представляет собой весьма трудную задачу.

\section{Список литературы}

1. Whitney $H$. On function with bounded $n$-th differences // J. Mat. Pure et Appl. 1955 . V. 36. P. 67-95.

2. Sendov B. On the constants of H. Whitney // C. R. Acad. Bul. Sci. 1982. V. 35. P. 424-431.

3. Брудный Ю.А. Об одной теореме локальных наилучших приближений // Уч. зап. Казанского ун-та. 1964. Т. 124. №6. С. 43-49.

4. Ivanov K., Takev M. $O(n \ln (n))$ bounds of constants of H. Whitney // C. R. Acad. Bul. Sci. 1985. V. 38. № 10. P. 1129-1131.

5. Binev P. $O(n)$ bounds of Whitney constants // C. R. Acad. Bul. Sci. 1985. V. 38. № 10. P. 1315-1317.

6. Sendov B. The constants of H.Whitney are bounded // C. R. Acad. Bul. Sci. 1985. V. 38. № 10. P. 1299-1303.

7. Sendov B. On the theorem and constants of H. Whitney // Constr. Approx. 1987. V. 3. P. 1-11.

8. Sendov B., Popov V. The average moduli of smoothness. Wiley \& Sons, 1988.

9. Брудный Ю. А. Константы Уитни: Тез. докладов. Современные проблемы теории функций. Баку, 1989.

10. Крякин Ю. В. О константах Уитни // Матем. заметки. 1989. Т. 46. № 2. С. 155-157.

11. Крякин Ю. В. О теореме и константах Уитни // Матем. сб. 1994. Т. 185. № 3. С. 25-40.

12. Стороженко Э.А. Приближение функций интерполяционными в среднем сплайнами // Изв. вузов. Математика. 1976. Т. 176. № 12. С. 82-95.

13. Kryakin Yu. V. On the theorem of H.Whitney in Spases $L_{p} / /$ Math. Balk. 1990. V. 4. № 3. P. 258-271.

14. Крякин Ю.В., Коваленко Л. Г. О теореме Уитни в $L_{p} / /$ Изв. вузов. Математика. 1992. №1. C. $69-77$. 
15. Sendov B., Takev M. The theorem of Whitney for integral norm // C. R. Acad. Bul. Sci. 1985. V. 38. № 10. P. 1199-1302.

16. Крякин Ю. В., Такев М. Д. Об интерполяционных константах Уитни // Матем. заметки. 1995.

17. Крякин Ю. В. О точных константах в теореме Уитни // Матем. заметки. 1993. Т. 54. № 1. C. $34-51$.

18. Брудный Ю. А. Многомерный аналог одной теоремы Уитни // Матем. сб. 1970. Т. 82. № 2. C. $175-191$.

19. Брудный Ю. А. Приближение функций $n$ переменных квазимногочленами // Изв. АН СССР. Сер. матем. 1970. Т. 34. №3. С. 568-585.

20. Стороженко Э. А. Приближение алгебраическими многочленами функций класса $L^{p}$, $0<p<1 / /$ Изв. АН СССР. Сер. матем. 1977. Т. 41. № 3. С. 652-662.

21. Стороженко Э. А., Освальд П. Теорема Джексонав пространствах $L^{p}\left(\mathbb{R}^{k}\right), 0<p<1$ // Сиб. матем. журнал. 1976. Т. 19. №4. С. 888-901.

22. Popov V., Petrushev P. Rational Approximation of Real Functions // Encyclopedia of Mathematics and its Applications. V. 28. Cambridge University Press, 1988.

23. Крякин Ю. В., Стороженко Э. А. О теореме Уитни в $L_{p}$-метрике // Матем. сб. 1995. № 3 .

24. Sendov B., Takev M. A Theorem of Whitney's type in $\mathrm{R}^{2} / /$ PLISKA Studia mathematica bulgarica. 1991. V. 11. P. 78-85.

25. Takev $M$. A theorem of Whitney type in $\mathbb{R}^{n} / /$ Constructive theory of functions '87. 1988 . P. 441-447. 